\title{
LAND USE LAND COVER MAPPING USING ADVANCED MACHINE LEARNING CLASSIFIERS
}

\author{
ALI JAMALI \\ Civil Engineering Department, Faculty of Engineering, Karabük Üniversitesi, Karabük, Turkey; e-mail: alijamali@karabuk.edu.tr; ali.jamali.65@gmail.com
}

Received: 8 March 2020 / Accepted: 29 May 2020

Abstract

Jamali A.: Land use land cover mapping using advanced machine learning classifiers. Ekológia (Bratislava), Vol. 40, No. 3, p. $286-300,2021$.

\begin{abstract}
Due to the recent climate changes such as floods and droughts, there is a need for Land Use Land Cover (LULC) mapping to monitor environmental changes that have effects on ecology, policy management, health and disaster management. As such, in this study, two well-known machine learning classifiers, namely, Support Vector Machine (SVM) and Random Forest (RF), are used for land cover mapping. In addition, two advanced deep learning algorithms, namely, the GAMLP and FSMLP, that are based on the Multi-layer Perceptron (MLP) function are developed in MATLAB programming language. The GAMLP uses a Genetic Algorithm (GA) to optimise parameters of the MLP function and, on the other hand, the FSMLP uses a derivative-free function for optimisation of the MLP function parameters. Three different scenarios using Landsat- 8 imagery with spatial resolutions of 30 and $15 \mathrm{~m}$ are defined to investigate the effects of data pre-processing on the final predicted LULC map. Results based on the statistical indices, including overall accuracy (OA) and kappa index, show that the developed MLP-based algorithms have relatively high accuracies with higher than $98 \%$ correct classification. Besides the statistical indices, final LULC maps are interpreted visually where the GAMLP and FSMLP give the best results for the pre-processed Landsat- 8 imagery with a spatial resolution of $15 \mathrm{~m}$, but they have the worst outcomes for the unprocessed Landsat- 8 imagery compared to SVM and RF classifiers visually and statistically.
\end{abstract}

Key words: LULC, machine learning, image classification, Multi-layer Perceptron, Support Vector Machine, Random Forest.

\section{Introduction}

In the last decade, remote sensing and data science researchers have used advanced machine learning algorithms for remote sensing image classification (Jamali, 2019; Mahdianpari et al., 2017, 2019; Rodriguez-Galiano et al., 2012; Rogan et al., 2008; Shao, Lunetta, 2012; Yeom et al., 2013). Free access satellite data such as Landsat- 8 and Sentinel- 2 has raised the use of image classification algorithms towards remote sensing field (Belward, Skøien, 2015; Harris, Baumann, 2015). On the other hand, the computing power of personal computers has been increasing, while its cost is decreasing at a rapid rate (Waldrop, 2016). In the Land Use Land Cover (LULC) mapping as a sub-field of image classification, the use of advanced machine learning algorithms has gained rapid interest (Jamali, 2020a,b,c; Jamali et al., 2021a,b) information on the Land Use Land Cover (LULC. For the physical and human environment, precise and up-to-data LULC dada is a need (Jamali, 2019), where it can be used in several fields, including health, ecology (Bourgeois, Sahraoui, 2020; Kenderessy et al., 2020; Skalský et al., 2020), policy management, agriculture and disaster management (Bégué et al., 2018).

Random Forest (RF) algorithm (Breiman, 2001) is considered as one of the most popular tree-based machine learning algorithms for image classification due to its simplicity and the fact that it can be used for both classification and regression problems with both continuous and categorical data (Woznicki et al., 2019). RF algorithms have been used in diverse fields such as land use modelling (Araki et al., 2018), forest cover mapping (Betts et al., 2017), land cover mapping (Nitze et al., 2017) and object-oriented mapping (Kavzoglu, 2017).

On the other hand, Support Vector Machine (SVM), due to its capability to generalise complex objects, has outperformed other machine learning classifiers in various researches (Mountrakis et al., 2011; Shao, Lunetta, 2012). For example, Goodin et al. (2015) used six land use classes to classify Landsat- 8 satellite images with the use of the SVM classifier, reaching a high overall accuracy (OA) of $88 \%$. Besides, Mansaray et al. (2020) used SVM and RF classifiers to map paddy rice in China in 2015 and 2016. In their study, SVM and RF obtained high accuracies of 90.8 and 89.2\%, respectively, for 2015 from Landsat- 8 and Sentinel-1A images. For 2016, RF and SVM classifiers had high accuracies of 95.2 and $93.4 \%$, respectively, from Landsat-8, Sentinel-1A and Sentinel-2A.

Additionally, from the late 1980s, neural networks have been intensively used for image classification in remote sensing, where they are utilised in open-source and commercial remote sensing software (Mas, Flores, 2008). Recently, deep learning classifiers are employed in various fields including land cover mapping ( $\mathrm{Li}$ et al., 2016), crop mapping (Kussul et al., 2017), plant disease mapping (Mohanty et al., 2016) and oil pam tree mapping ( $\mathrm{Li}$ et al., 2017). In this research, besides, two well-known machine learning algorithms, including SVM and RF classifiers, two ad-

(C) The Author(s) 2021. This is an open access article distributed under the terms of the CC BY-NC-ND license.

https://content.sciendo.com/view/journals/eko/eko-overview.xml 

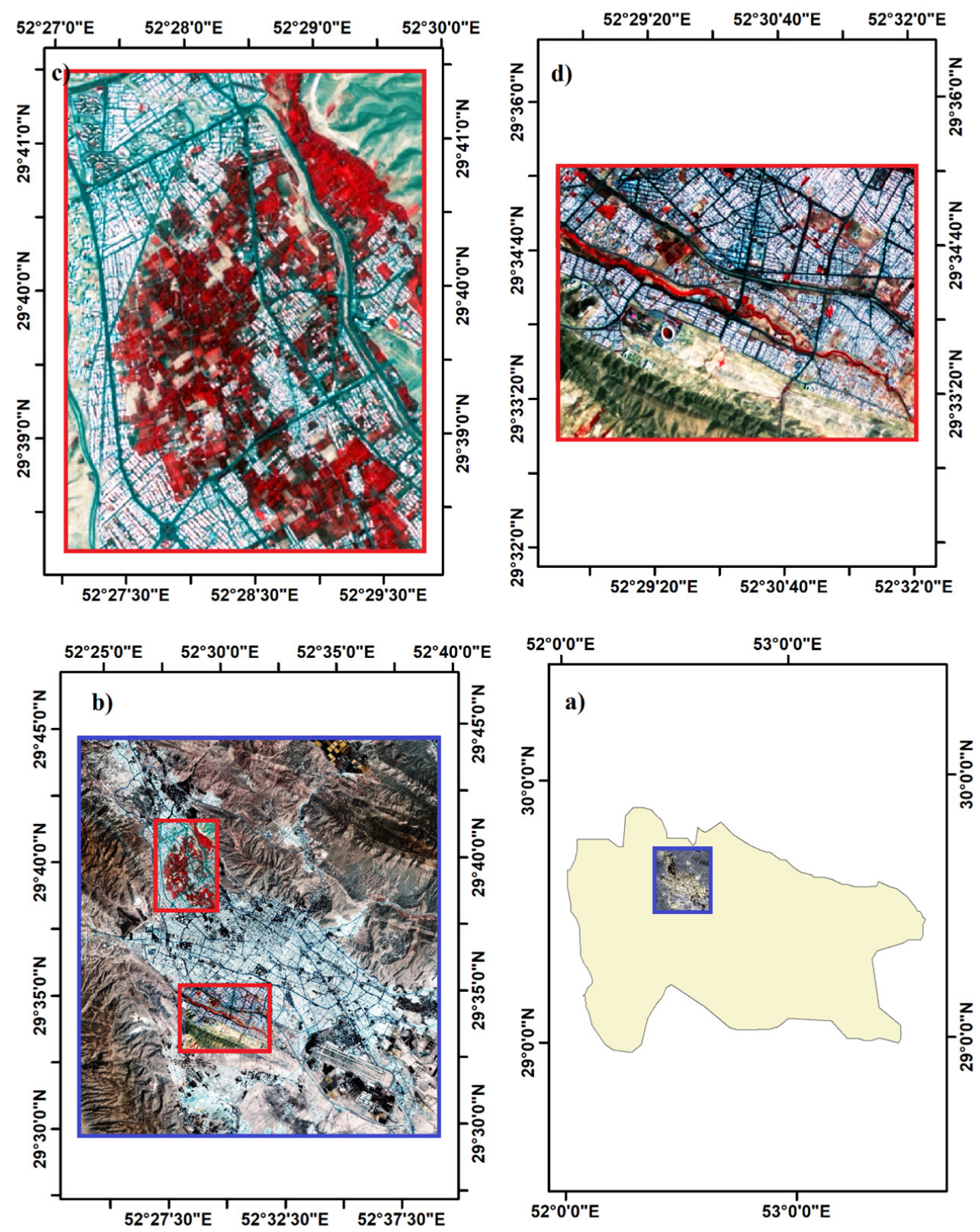

Fig. 1. The study area. a) Fars province; b) Shiraz city; c) region a in false colour; d) region b in false colour.

vanced classifiers, namely, the derivative-free function of FminSearch Multi-Layer Perceptron (FSMLP) and Genetic Algorithm Multi-Layer Perceptron (GAMLP), are developed in the MATrix LABoratory (MATLAB) programming language. In addition, results are compared in terms of OA and kappa index, and they are interpreted visually for various regions of the study area.

\section{Methods}

\section{Study area and data collection}

The study area was Shiraz city, which is situated in Fars province of Iran (see Fig. 1a,b). For visual interpretation of the resulting
LULC maps, two regions of $6 \mathrm{~km}$ by $4.5 \mathrm{~km}$ (see Fig. $1 \mathrm{c}$ ) and 4.5 by $6 \mathrm{~km}$ (see Fig. 1d) were selected. In region a, garden areas are a dominant class, whereas in region $b$, urban and soil areas are dominant classes.

Shiraz city is chosen as there is a huge number of gardens in the city. Most of the gardens are lost due to climate change (i.e., droughts) and recent rapid urbanisation. The researcher seeks for a machine learning algorithm for the monitoring of the city which can help with the preservation of the garden regions in the study area.

The multi-spectral Landsat-8 Optical Land Imager (OLI) image was acquired on 22 August 2018 and downloaded from the Earth Resources Observation and Science Centre of the US Geo- 
Table 1. Description of the seven Landsat- 8 bands used in this study.

\begin{tabular}{|l|l|l|l|}
\hline Band & Spectral region & SR $(\mathbf{m})$ & $\mathbf{C W}(\boldsymbol{\mu m})$ \\
\hline $\mathbf{1}$ & Coastal aerosol & 30 & 0.4430 \\
\hline $\mathbf{2}$ & Blue & 30 & 0.4826 \\
\hline $\mathbf{3}$ & Green & 30 & 0.5613 \\
\hline $\mathbf{4}$ & Red & 30 & 0.6546 \\
\hline $\mathbf{5}$ & NIR & 30 & 0.8646 \\
\hline $\mathbf{6}$ & SWIR 1 & 30 & 1.6090 \\
\hline $\mathbf{7}$ & SWIR 2 & 30 & 2.2010 \\
\hline
\end{tabular}

Notes: SR - spatial resolution, CW - central wavelength, NIR - near infrared.

Table 2. Total number of pixels in each sample and the number of pixels selected for the training and evaluation.

\begin{tabular}{|l|c|c|c|}
\hline LULC type & Class ID & Total pixels & $\begin{array}{c}\text { Samples } \\
\text { (evaluation/training) }\end{array}$ \\
\hline Vegetation & 1 & 1112 & $779 / 333$ \\
\hline Urban & 2 & 211 & $148 / 63$ \\
\hline Soil & 3 & 12,364 & $8655 / 3709$ \\
\hline Roads & 4 & 461 & $323 / 138$ \\
\hline
\end{tabular}

logical Survey. The multi-spectral Landsat- 8 has a spatial resolution of $30 \mathrm{~m}$ and consists of seven bands with a wavelength from 0.4430 to $2.2010 \mu \mathrm{m}$ (see Table 1 ).

The Landsat- 8 image is in a Level- 2 format, which means that it is a geometrically corrected image. To compare and investigate the effect of atmospheric correction and pan-sharpening on LULC mapping, three different scenarios are created. These scenarios may help with the understanding of the best-suited data for image classification for the future use and how image pre-processing affects the predicted LULC maps. corrected and bands are pan-sharpened to a spatial resolution of $15 \mathrm{~m}$ with the use of Gram-Schmidt pan-sharpening algorithm in the Environment for Visualizing Images (ENVI) software. From seven multi-spectral bands, three spectral indices including Normalised Difference Vegetation Index (NDVI), Normalised Difference Built-up Index (NDBI) and Simple Ratio (SR) are derived. NDVI presents the ratio between energy absorbed by the vegetation in the red band and the reflected energy in the near-infrared (NIR) band (Rouse et al., 1973). The Short Wave Infra-Red (SWIR) band is used in NDBI to detect artificial surfaces due to their strong reflectivity (Zha et al., 2003). The SR helps with the identification of vegetation due to the high reflectance of vegetation in the NIR band and high absorption in the red band. In scenario 2, seven multi-spectral bands of Landsat- 8 are utilised without any correction at a spatial resolution of 30 $\mathrm{m}$. In scenario 3, Landsat-8 image has been atmospherically corrected and bands are pan-sharpened to a spatial resolution of 15 $\mathrm{m}$ with the use of Gram-Schmidt pan-sharpening algorithm in ENVI software. In addition, Landsat-8 image is segmented using a multi-resolution segmentation algorithm with a scale factor of 15 , a shape factor of 0.5 and a compactness factor of 0.5 in eCognition software.
In scenario 1, Landsat-8 image has been atmospherically

\section{Study design and sample selection}

Figure 2 presents the flowchart of the methods used in this research.In this research, there are 4243 pixels for training samples and 9905 pixels for test samples. There are four LULC types: vegetation, urban, soil and roads (see Table 2).

\section{Machine learning classifiers}

\section{Support Vector Machine}

Based on the work of Vapnik (1982), Cortes and Vapnik (1995) described SVM as a supervised machine learning algorithm which is one of the most utilised algorithms by remote sensing communities. The SVM algorithm searches for the optimal minimisation (decision boundary) of unknown classifier outputs in a problem space. To find the patterns between training data, SVM with the use of an iterative process in an n-dimensional space searches for the optimal hyperplane boundary, and it applies the same parameters to the validation samples. According to Mountrakis et al. (2011), in the field of remote sensing, the dimensions are defined by the number of spectral bands, while vectors are defined by the pixels.

\section{Random Forest}

Considering that a combination of bootstrap-integrated classifiers gives better results than a single classifier, RF is defined as an ensemble learning algorithm (Breiman, 2001). With the use of a random sample set of observations, each tree is parameterised, where they are replaced by training data samples (Hastie et al., 2009). Out-of-bag samples are the observations that are not used in the process, and they are used as evaluation samples. Several of these decision tree models are created on different groupings of the input variables. The final model (i.e., optimal model) is generated using majority voting of all trees for each class.

\section{Genetic Algorithm Multi-Layer Perceptron and fmin- search Multi-Layer Perceptron}

One of the most advanced heuristic search algorithms in machine learning is Genetic Algorithm (GA), which has been widely used in various applications such as ecology, urban planning and remote sensing (Chang et al., 2006; Chen et al., 2009; Hasegawa et al., 2006). A GA can be described as a global optimisation method that is not dependent on the initial values.

In this research, in the Multi-layer Perceptron (MLP) algorithm, each output (i.e., class) is calculated using a hyperbolic tangent sigmoid transfer function (see Equations 1 and 2). For all three scenarios, three hidden neurons were selected experimentally. For scenarios 2 and 3, the number of inputs is 7, and the number of inputs for scenario 1 is 10 .

$$
\begin{gathered}
a_{i}=\operatorname{TANGSIG}\left(\left(w_{h} \times x_{i}\right)+b_{h}\right) \\
o_{i}=\left(w_{o} \times \alpha_{i}\right)+b_{o} \\
w_{h}=\left[\begin{array}{ccc}
w h_{h_{11}} & \cdots & w h_{1 i n} \\
\vdots & \ddots & \vdots \\
w h_{31} & \cdots & w h_{3 i n}
\end{array}\right]_{3 \times i n}, b_{h}=\left[\begin{array}{c}
b h_{1} \\
\vdots \\
b h_{3}
\end{array}\right]_{3 \times 1}, w_{o}=\left[\begin{array}{lll}
w o_{1} & \cdots & w o_{h}
\end{array}\right]_{1 \times 3}, b_{o}=b o, x_{i}=\left[\begin{array}{c}
x_{1} \\
\vdots \\
x_{i n}
\end{array}\right]_{i n \times 1} .
\end{gathered}
$$




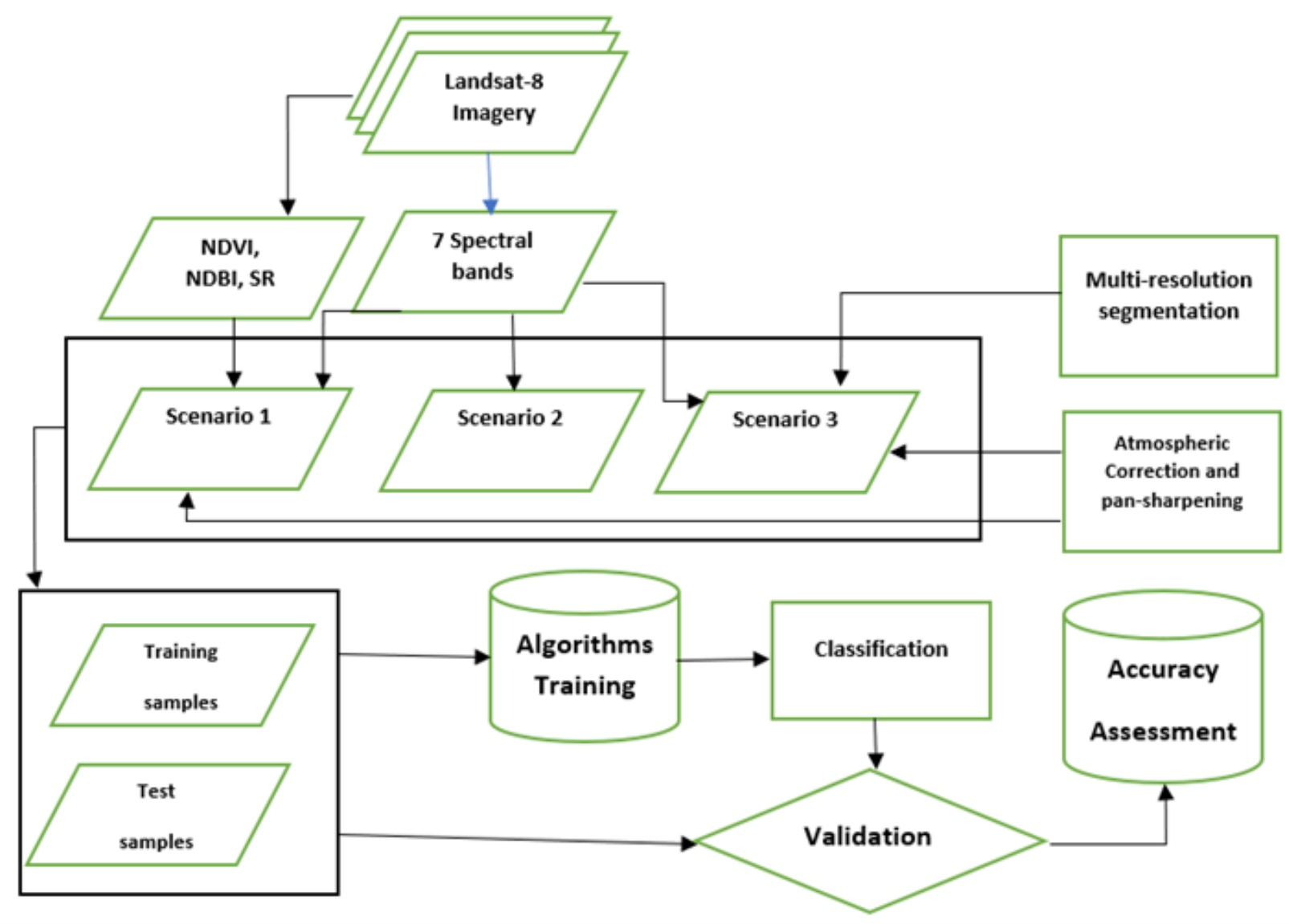

Fig. 2. Flowchart of the methods.

Notes: NDVI = Normalised Difference Vegetation Index; NDBI = Normalised Difference Built-up Index; SR = Simple Ratio.

where TANGSIG is the hyperbolic tangent sigmoid transfer function, is the weight of the hidden neuron, is the bias of hidden neuron, is the weight of output and is the bias of output. is the class of the training point $\mathrm{i}, \mathrm{i}=1,2, \ldots, 4243$. is the inputs $(7$ for scenarios 2 and 3, 10 for scenario 1 ) for training point i. Considering seven bands for scenarios 2 and 3, three hidden neurons and an output, the architecture of the MLP method is presented in Figure 3. As a result, there are 28 connections (coefficients) to be approximated for scenarios 2 and 3.

There are six steps for developing two optimised machine learning algorithms (the GAMLP and FSMLP) based on the MLP function, which are as follows (see Fig. 4):

1. Data preparation: In this step, 4243 training samples are divided into training (70\%) and validation (30\%) datasets.

2. Initial model generation: In this step, with the help of Neural Fitting Toolbox in MATLAB programming language, initial populations, which are 28 and 37 coefficients for scenarios $1-3$, are approximated.

3. Metaheuristic optimisation: In this step, GA and fminsearch, which is a derivative-free function, are used to optimise parameters of the developed MLP function. For the GA, a hybrid function (fminunc Unconstrained Minimisation) is used. Considering that error is the total number of misclassified training samples after GA reached its stopping criteria, fminunc searches for the minimum of error mathematically described as .
4. Model evaluation: In this step, the error is used to evaluate the performance of the MLP function.

5. Stopping criteria: The optimisation process will continue till the generation of 100 times the number of variables $(2800$ for scenarios 2 and 3, 3700 for scenario 1).

6. Final optimised models: In this step, the final GAMLP and FSMLP are generated using the best parameters with the minimum error. It is worth highlighting that the developed models are validated using their total number of misclassified training, validation and test samples.

With the use of fminsearch, 28 coefficients for scenarios 2 and 3 and 37 coefficients for scenario 1 are optimised using a derivative-free method based on the error-index, which is the total number of misclassified classes of the 4243 training pixels. The derivative-free method searches for the minimum of error using training data described mathematically as (see Equation 3):

$$
\left.\min _{\text {Error }} f \text { (coefficients, input } t_{\text {train }}, \text { class }_{\text {train }}\right)
$$

\section{Model training and hyper-parameter optimisation}

The SVM and RF classifiers are implemented in R programming language with the use of the caret library. Using a 10-repeated 10 -fold cross-validation method, models are optimised with the 


\section{$\mathrm{B}=\mathrm{Bands}$}

\section{$\mathrm{BH}=\mathrm{Bias}$ of hidden neurons}

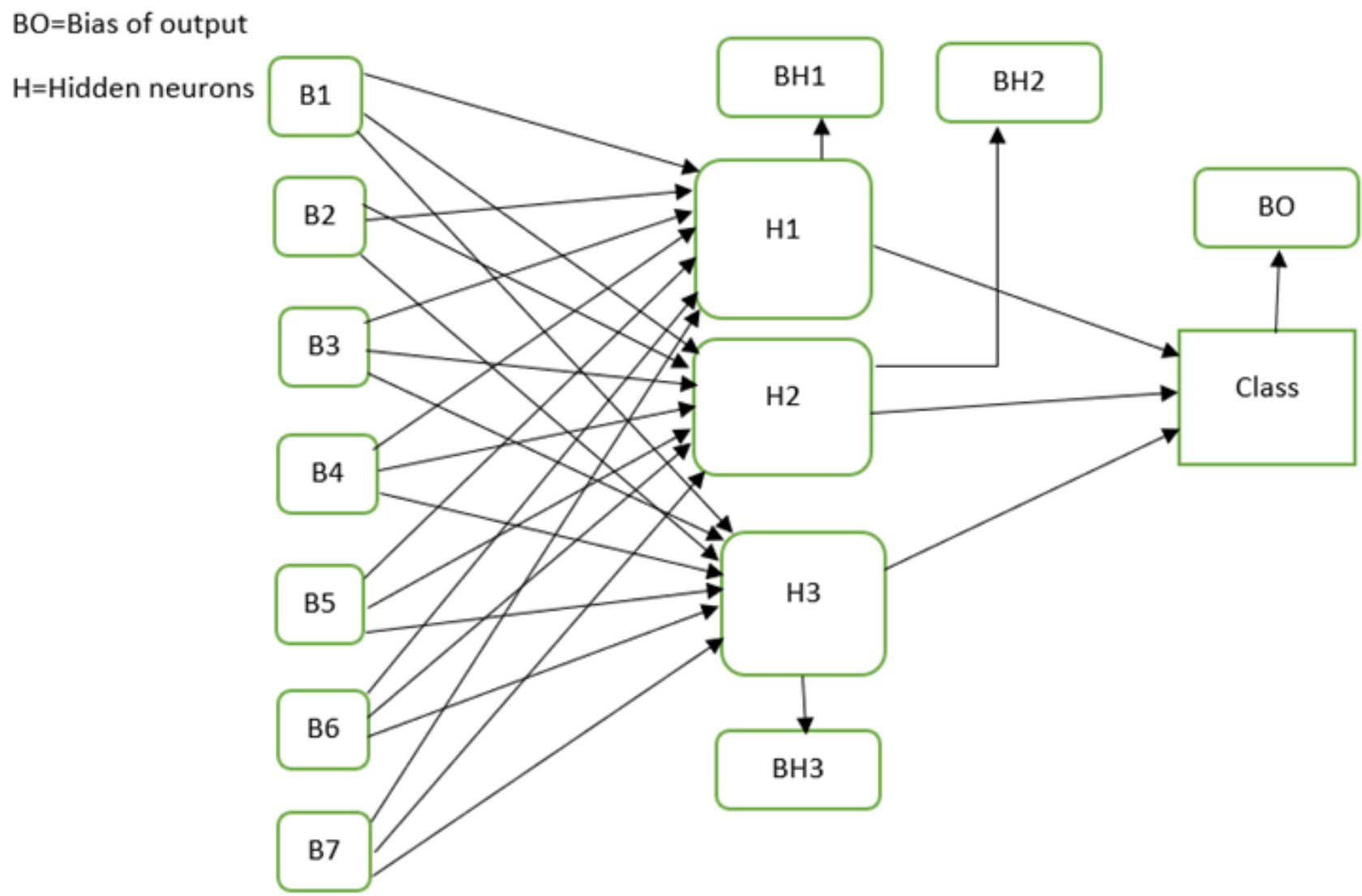

Fig. 3. Architecture of the MLP.

Note: MLP - Multi-layer Perceptron.

Table 3. Results of the hyper-parameter optimisation process showing the final values used in each model. The tuned length was set to 1000 iterations.

\begin{tabular}{|l|l|l|}
\hline Model & Hyper-parameter value & Definition \\
\hline SVM & $\begin{array}{l}\text { Kernel }=\text { 'rbf' } \\
\text { sigma }=0.01328357 \text { and C }=1 \text { for scenario 1 } \\
\text { sigma }=1.535998 \text { and C }=0.5 \text { for scenario 2 } \\
\text { sigma }=1.413116 \text { and } C=0.25 \text { for scenario 3 }\end{array}$ & $\begin{array}{l}\mathrm{C}=\text { cost of misclassification. } \\
\text { RBF = radial basis function, a multidimensional Gaussian distribu- } \\
\text { tion function is describing the distance between an input vector and a } \\
\text { pre-defined centre vector. sigma = weight of the RBF kernel }\end{array}$ \\
\hline RF & $\begin{array}{l}\text { mtry = number of variables randomly sampled as candidates at each } \\
\text { split }\end{array}$ \\
\hline
\end{tabular}

Notes: SVM - Support Vector Machine; RF - Random Forest.

hyper-parameter tuning method. Optimal hyper-parameters are selected based on the OA index. Model optimisation for SVM and RF algorithms is done using a 1000 number of iterations based on the normalised sampling of combinations of all hyper-parameters (Kuhn, 2008). Table 3 presents the results of the hyper-parameter optimisation values for all the three defined scenarios.

\section{Accuracy assessment}

The OA and kappa indices were used to evaluate the accuracy of each machine learning method. Figures 5 and 6 present the results of machine learning accuracies for the validation and test samples. There were four predicted images in each scenario, resulting in 12 final predicted LULC maps. For the validation sam- 


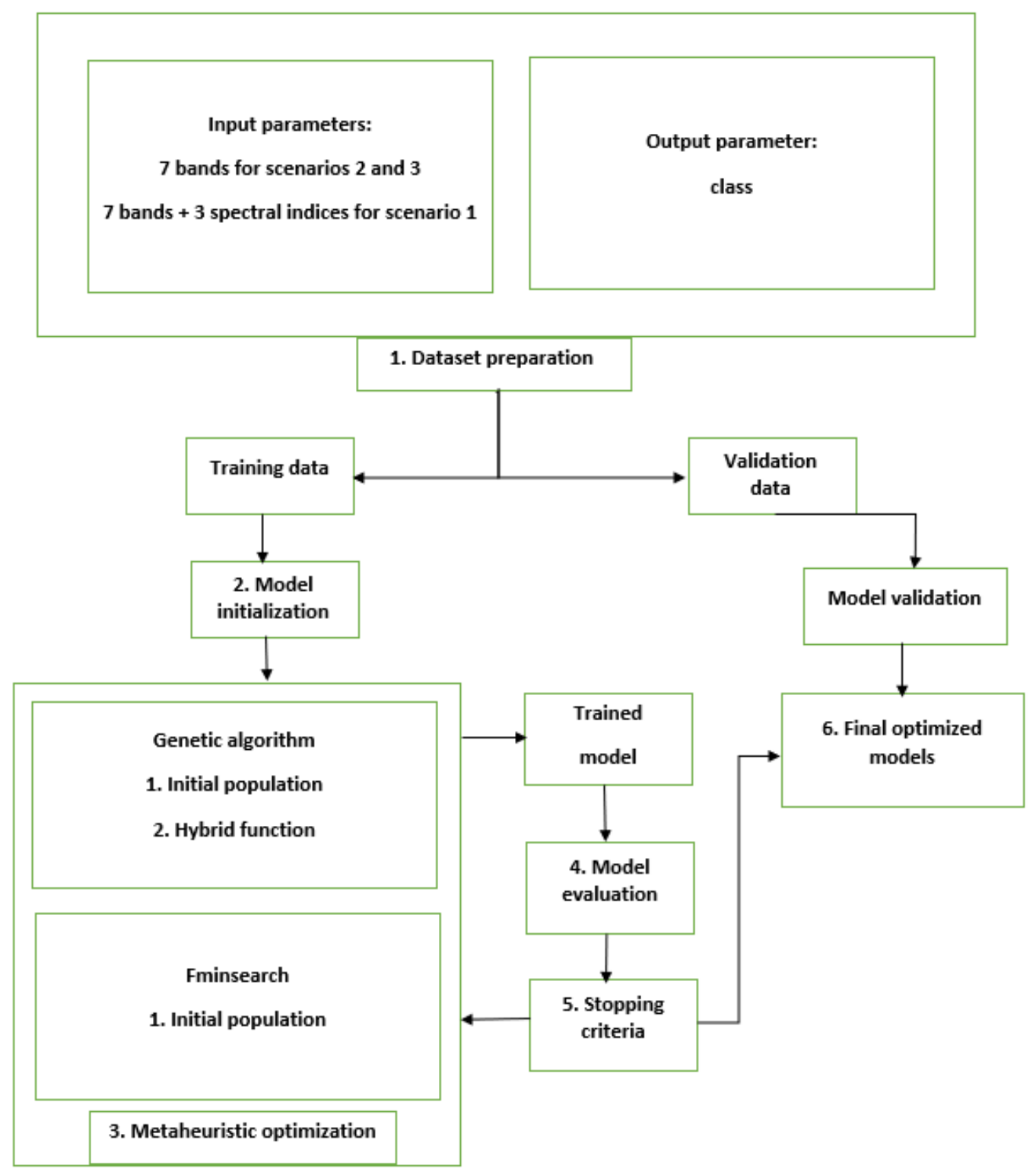

Fig. 4. Flowchart of the developed models.

ples, GAMLP15px, FSMLP15px from scenario 1 and SVM15ob, GAMLP15ob, FSMLP15ob from scenario 3 had perfect OA and kappa values with zero misclassified samples. Moreover, RF30px from scenario 2 had the worst result with values of 0.9981148 and 0.99176 for the OA and Kappa indices, respectively (see Fig. 5).

Based on the test samples, FSMLP15ob and GAMLP15ob from scenario 3 had perfect results without any misclassified samples. In addition, FSMLP30px and GAMLP30px showed the worst results with the values of 0.9960 and 0.9827 for the OA and Kappa indices, respectively (see Fig. 6).

Table 4 presents the error matrices showing correct and incorrect cross-tabulations of the test samples by each machine learning algorithm.

\section{Results and discussion}

The developed machine learning algorithms produced different LULC maps for the three scenarios defined (Figs 8-13). Final maps for the study area region a are visually interpreted based on the four regions in colours, including two urban areas (red and white colour), bare soil space (yellow colour) and a junction (orange colour). Final maps for the study area in region $b$ are visually interpreted based on the two regions in colours, including a stadium (pink colour) and bare soil area (purple colour) (see Fig. 7).

For the study area in region a, in scenario 1 with a spatial resolution of $15 \mathrm{~m}$, the SVM, FSMLP and GAMLP classifiers 


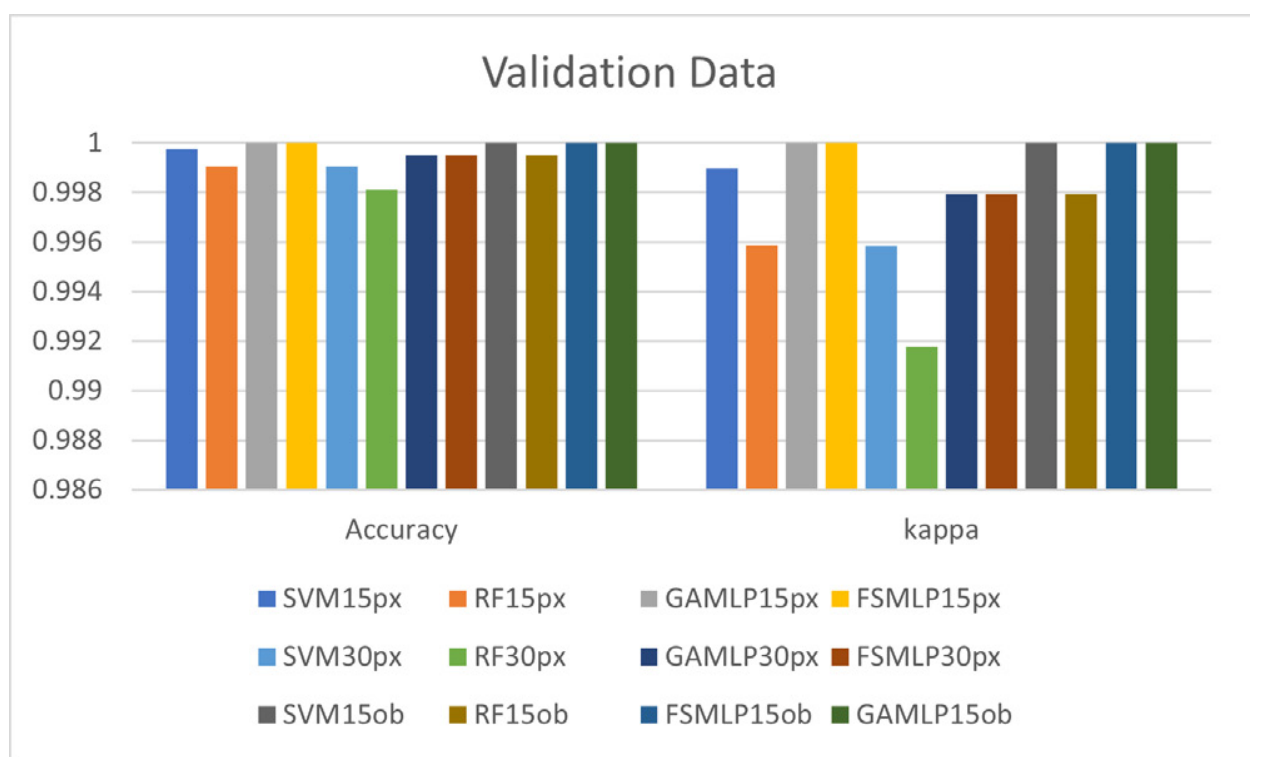

Fig. 5. Results of machine learning algorithms for the validation dataset. 15px are images in scenario 1, 15ob are images in scenario 3 and $30 \mathrm{px}$ are images in scenario 2 .

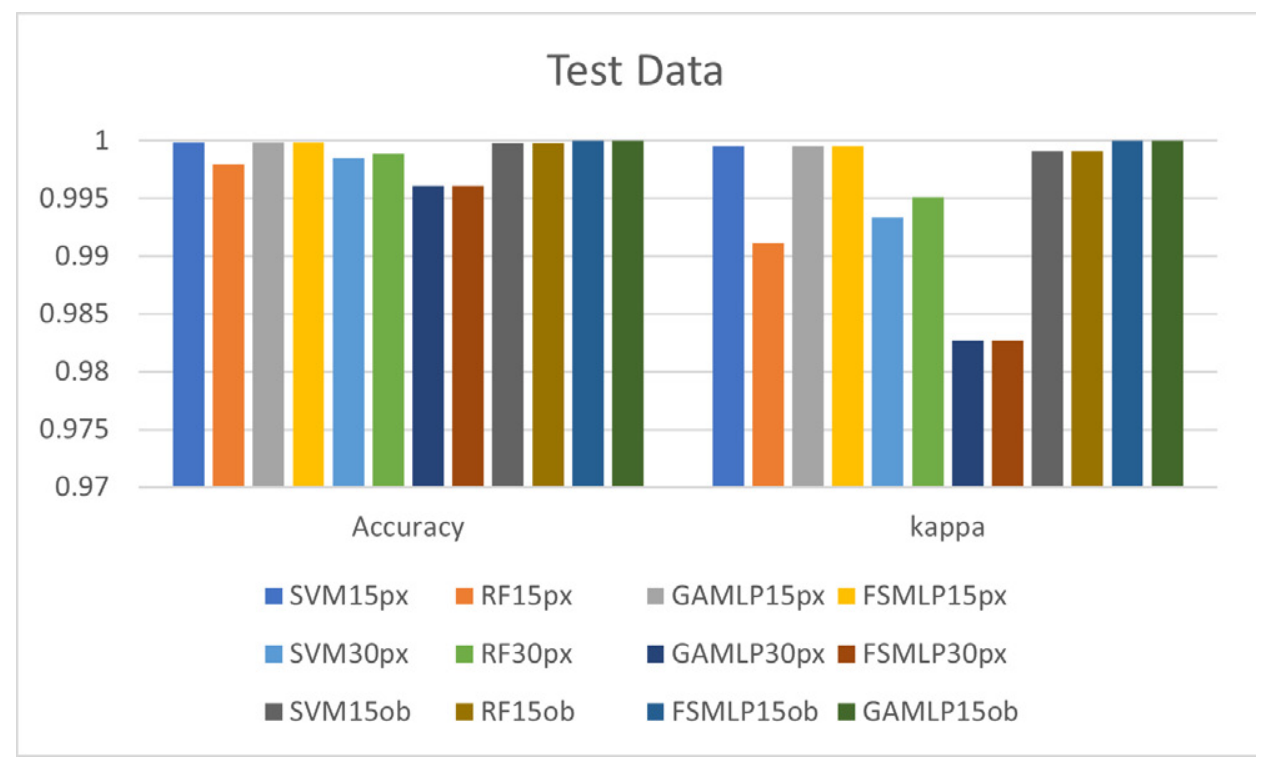

Fig. 6. Results of machine learning algorithms for the test dataset. 15px are images in scenario 1, 15ob are images in scenario 3 and $30 \mathrm{px}$ are images in scenario 2 .

presented relatively good results visually. However, the RF method had a speckled map with the worst result compared to the other machine learning classifiers (see Fig. 8). All four machine learning algorithms classified the roads well.

For the study area in region a, in scenario 3 with a spatial resolution of $15 \mathrm{~m}$, the SVM, FSMLP and GAMLP algorithms presented relatively good results visually. The RF classifier had a speckled map with the worst outcome (see Fig. 9). Moreover, the GAMLP and SVM classifiers classified road regions better compared to those of the RF and FSMLP algorithms.
For the study area in region a, in scenario 2 with a spatial resolution of $30 \mathrm{~m}$, the SVM and RF algorithms presented relatively good results compared to the other MLP-based methods. The GAMLP and FSMLP classifiers had produced speckled maps with the worst results for urban recognition (see Fig. 10). All four machine learning classifiers gave relatively good results for the identification of road regions.

For the study area in region $b$, in scenario 1 with a spatial resolution of $15 \mathrm{~m}$, the SVM, FSMLP and GAMLP algorithms showed relatively good results. The RF method had a speckled map with the worst result visually that produced lots of mis- 
classified urban areas (see Fig. 11). In addition, the RF classifier failed at road and vegetation regions recognition compared to the other supervised machine learning approaches.

For the study area in region $b$, in scenario 3 with a spatial resolution of $15 \mathrm{~m}$, the FSMLP and GAMLP algorithms presented relatively good results visually. The RF method had the worst results visually with lots of misclassified urban and vegetation areas (see Fig. 12). Notably, none of the classifiers could predict road regions with an acceptable accuracy visually. In addition, the SVM classifier did not produce good results for the identification of urban and vegetation regions.

For the study area in region $b$, for scenario 2 with a spatial resolution of $30 \mathrm{~m}$, the RF algorithm showed relatively good results for the recognition of all four classes compared to the other classifiers visually. The FSMLP and GAMLP methods had speckled maps (see Fig. 13). The SVM and RF classifiers presented relatively good results for urban, soil and road region identification. Besides, the SVM algorithm did not produce good results for the recognition of vegetation areas.

\section{Conclusion}

The main objective of this research was to fulfil the need for a well-suited advanced algorithm to monitor garden regions in Shiraz city. In addition, to investigate the best spatial resolution for the research objective, three different scenarios with a spatial resolution of 15 and $30 \mathrm{~m}$ were defined. Results showed that all machine learning algorithms predicted the four classes of urban, soil, road, and vegetation with accuracies higher than $98 \%$. For visual interpretation of the predicted LULC maps, several regions were compared visually, as well.

The developed GAMLP and FSMLP algorithms, which were based on the MLP function, gave good results statistically and visually for the pre-processed Landsat- 8 imagery, whereas they did not produce good results for the unprocessed image compared to SVM and RF classifiers. The SVM classifier showed relatively consistent results for both preprocessed and unprocessed Landsat- 8 imagery, whereas the tree-based RF algorithm failed in the prediction of the LULC classes compared to the other three machine learning methods. Finally, based on the results, it was concluded that the GAMLP and FSMLP methods were reliable classifiers for monitoring the study area where Landsat- 8 imagery was preprocessed and they had a medium resolution of $15 \mathrm{~m}$.

\section{References}

Araki, S., Shima, M. \& Yamamoto K. (2018). Spatiotemporal land use random forest model for estimating metropolitan NO2 exposure in Japan. Sci. Total Environ., 634, 1269-1277. DOI: 10.1016/j. scitotenv.2018.03.324.

Bégué, A., Arvor, D., Bellon, B., Betbeder, J., de Abelleyra, D., Ferraz, R.P.D., Lebourgeois, V., Lelong, C., Simőes, M. \& Verón S.R. (2018). Remote sensing and cropping practices: A review. Remote Sensing, 10, 99. DOI: 10.3390/rs10010099.

Belward, A.S. \& Skøien J.O. (2015). Who launched what, when and why; trends in global land-cover observation capacity from civilian earth observation satellites. ISPRS Journal of Photogrammetry and Remote Sensing, 103, 115-128. DOI: 10.1016/j.isprsjprs.2014.03.009.

Betts, M.G., Christopher Wolf, W.J., Ripple, B.P., Millers, K.A., Adam Duarte, S.H., Butchart, M. \& Levi T. (2017). Global forest loss disproportionately erodes biodiversity in intact landscapes. Nature, 547, 441-447. DOI: 10.1038 /nature23285.
Table 4. Error matrices showing correct and incorrect cross-tabulations of the test samples by each machine learning algorithm. The LCLU classes are numbered 1 through 4 , where $1=$ vegetation, $2=$ urban, $3=$ soil and $4=$ roads. 15 px are images in scenario $1,15 \mathrm{ob}$ are images in scenario 3 and $30 \mathrm{px}$ are images in scenario 2.

\begin{tabular}{|c|c|c|c|c|c|c|c|c|c|}
\hline \multicolumn{5}{|c|}{ SVM15px } & \multicolumn{5}{|c|}{ RF15ob } \\
\hline & 1 & 2 & 3 & 4 & & 1 & 2 & 3 & 4 \\
\hline 1 & 779 & 0 & 0 & 0 & 1 & 779 & 0 & 0 & 0 \\
\hline 2 & 0 & 147 & 0 & 0 & 2 & 0 & 146 & 0 & 0 \\
\hline 3 & 0 & 0 & 8655 & 0 & 3 & 0 & 2 & 8655 & 0 \\
\hline 4 & 0 & 1 & 0 & 323 & 4 & 0 & 0 & 0 & 323 \\
\hline \multicolumn{5}{|c|}{ GAMLP30px } & \multicolumn{5}{|c|}{ SVM15ob } \\
\hline & 1 & 2 & 3 & 4 & & 1 & 3 & 3 & 4 \\
\hline 1 & 769 & 0 & 0 & 5 & 1 & 779 & 0 & 0 & 0 \\
\hline 2 & 2 & 145 & 4 & 7 & 2 & 0 & 146 & 0 & 0 \\
\hline 3 & 6 & 3 & 8651 & 10 & 3 & 0 & 2 & 8655 & 0 \\
\hline 4 & 2 & 0 & 0 & 301 & 4 & 0 & 0 & 0 & 323 \\
\hline \multicolumn{5}{|c|}{ FSMLP30px } & \multicolumn{5}{|c|}{ RF30px } \\
\hline & 1 & 2 & 3 & 4 & & 1 & 2 & 3 & 4 \\
\hline 1 & 769 & 0 & 0 & 5 & 1 & 777 & 0 & 0 & 0 \\
\hline 2 & 2 & 145 & 4 & 7 & 2 & 0 & 141 & 0 & 2 \\
\hline 3 & 6 & 3 & 8651 & 10 & 3 & 1 & 1 & 8655 & 0 \\
\hline 4 & 2 & 0 & 0 & 301 & 4 & 1 & 6 & 0 & 321 \\
\hline \multicolumn{5}{|c|}{ GAMLP15ob } & \multicolumn{5}{|c|}{ SVM30px } \\
\hline & 1 & 2 & 3 & 4 & & 1 & 2 & 3 & 4 \\
\hline 1 & 779 & 0 & 0 & 0 & 1 & 777 & 0 & 0 & 0 \\
\hline 2 & 0 & 148 & 0 & 0 & 2 & 0 & 143 & 0 & 9 \\
\hline 3 & 0 & 0 & 8655 & 0 & 3 & 2 & 1 & 8655 & 0 \\
\hline 4 & 0 & 0 & 0 & 323 & 4 & 0 & 4 & 0 & 314 \\
\hline \multicolumn{5}{|c|}{ FSMLP15ob } & \multicolumn{5}{|c|}{ RF15px } \\
\hline & 1 & 2 & 3 & 4 & & 1 & 2 & 3 & 4 \\
\hline 1 & 779 & 0 & 0 & 0 & 1 & 779 & 0 & 0 & 0 \\
\hline 2 & 0 & 148 & 0 & 0 & 2 & 0 & 137 & 3 & 0 \\
\hline 3 & 0 & 0 & 8655 & 0 & 3 & 0 & 11 & 8652 & 6 \\
\hline 4 & 0 & 0 & 0 & 323 & 4 & 0 & 0 & 0 & 317 \\
\hline \multicolumn{5}{|c|}{ FSMLP15px } & \multicolumn{5}{|c|}{ GAMLP15px } \\
\hline & 1 & 2 & 3 & 4 & & 1 & 2 & 3 & 4 \\
\hline 1 & 779 & 0 & 0 & 0 & 1 & 779 & 0 & 0 & 0 \\
\hline 2 & 0 & 148 & 0 & 1 & 2 & 0 & 148 & 0 & 0 \\
\hline 3 & 0 & 0 & 8655 & 0 & 3 & 0 & 0 & 8655 & 1 \\
\hline 4 & 0 & 0 & 0 & 322 & 4 & 0 & 0 & 0 & 322 \\
\hline
\end{tabular}

Bourgeois, M. \& Sahraoui Y. (2020). Modelling in the context of an environmental mobilisation: a graph-based approach for assessing the landscape ecological impacts of a highway project. Ekológia (Bratislava), 39(1), 88-100. DOI: 10.2478/eko-2020-007.

Breiman, L. (2001). Random forests. Machine Learning, 45(1), 5-32. DOI: 10.1023/a:1010933404324.

Chang, C., Lo, S. \& Yu S. (2006). The parameter optimization in the inverse distance method by genetic algorithm for estimating precipitation. Environ. Monit. Assess., 117, 145-155. DOI: 10.1007/s10661-006-8498-0.

Chen, Y., Chen, J., Hsieh, S. \& Ni P. (2009). The application of remote sensing technology to the interpretation of land use for rainfall-induced landslides based on genetic algorithms and artificial neural networks. IEEE Journal of Selected Topics in Applied Earth Observations and Remote Sensing, 2, 87-95. 

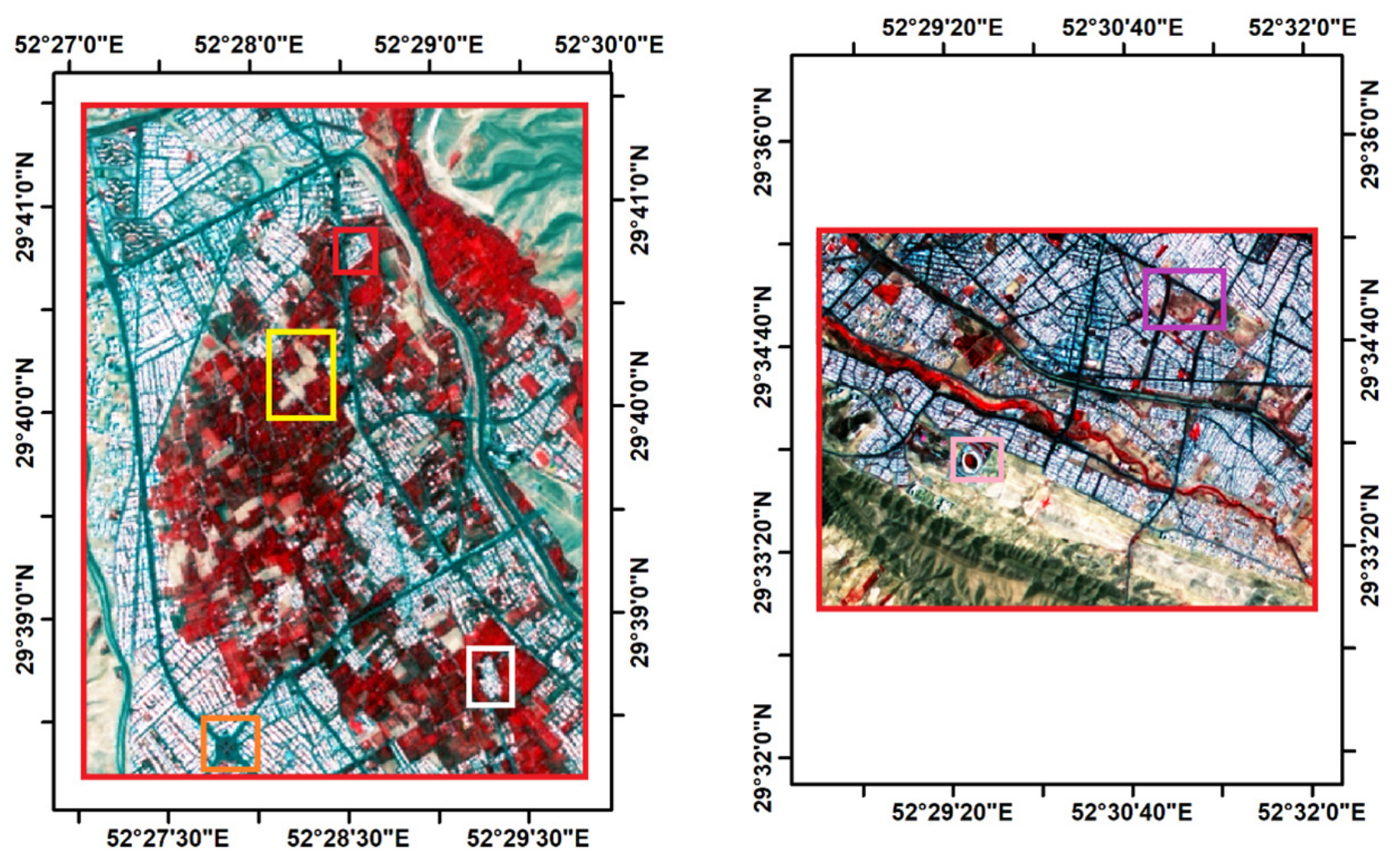

Fig. 7. a) Four regions of the study area in region a (left image); b) two areas of the study area in region b (right image).

Cortes, C. \& Vapnik V. (1995). Support-vector networks. Machine Learning, 20(3), 273-297. DOI: 10.1007/bf00994018.

Goodin, D.G., Anibas, K.L. \& Bezymennyi M. (2015). Mapping land cover and land use from object-based classification: An example from a complex agricultural landscape. Int. J. Remote Sens., 36(18), 4702-4723. DOI: 10.1080/01431161.2015.1088674.

Harris, R. \& Baumann I. (2015). Open data policies and satellite earth observation. Space Policy, 32, 44-53. DOI: 10.1016/j.spacepol.2015.01.001.

Hasegawa, H., Arimura, M. \& Tamura T. (2006). Hybrid model of random forests and genetic algorithms for commute mode choice. Analysis, 9.

Hastie, T., Tibshirani, R. \& Friedman J. (2009). Random forests. In The elements of statistical learning: Data mining, inference, and prediction (pp. 587-604) New York: Springer. DOI: 10.1007/978-0-387-84858-7_15.

Jamali, A. (2019). Evaluation and comparison of eight machine learning models in land use/land cover mapping using Landsat 8 OLI: a case study of the northern region of Iran. SN Applied Sciences, 1, 1448. DOI: 10.1007/s42452-019-1527-8.

Jamali, A. (2020a). Improving land use land cover mapping of a neural network with three optimizers of multi-verse optimizer, genetic algorithm, and derivative-free function. The Egyptian Journal of Remote Sensing and Space Science. DOI: 10.1016/j.jejrs.2020.07.001.

Jamali, A. (2020b). Land use land cover mapping using advanced machine learning classifiers: A case study of Shiraz city, Iran. Earth Science Informatics. DOI: 10.1007/s12145-020-00475-4.

Jamali, A. (2020c). Land use land cover modeling using optimized machine learning classifiers: a case study of Shiraz, Iran. Model. Earth Syst. Environ. DOI: 10.1007/s40808-020-00859-x.

Jamali, A., Mahdianpari, M., Brisco, B., Granger, J., Mohammadimanesh, F. \& Salehi B. (2021a). Comparing Solo Versus Ensemble Convolutional Neural Networks for Wetland Classification Using Multi-Spectral Satellite Imagery. Remote Sensing, 13(11), 2046. DOI: 10.3390/rs13112046.
Jamali, A., Mahdianpari, M., Brisco, B., Granger, J., Mohammadimanesh, F. \& Salehi B. (2021b). Wetland Mapping Using Multi-Spectral Satellite Imagery and Deep Convolutional Neural Networks: A Case Study in Newfoundland and Labrador, Canada. Canadian Journal of Remote Sensing, 1-18. DOI: 10.1080/07038992.2021.1901562.

Kavzoglu, T. (2017). Object-oriented random forest for high resolution land cover mapping using quickbird-2 imagery. In S.S.P. Samui, S. Sekhar \& V.E. Balas (Eds.), Handbook of neural computation (pp. 607-619). Cambridge: Academic Press. DOI: 10.1016/b978-0-12-811318-9.00033-8.

Kenderessy P., Kollár, J. \& Palaj A. (2020). The impact of historical agricultural landuse on selected site conditions in the traditional landscape of the West Carpathians. Ekológia (Bratislava), 39(4), 343-356. DOI: 10.2478/ eko-2020-0028.

Kuhn, M. (2008). Building Predictive Models in R Using the caret Package. Journal of Statistical Software, 1(5). https://www.jstatsoft.org/v028/i05

Kussul, N., Lavreniuk, M., Skakun, S. \& Shelestov A. (2017). Deep learning classification of land cover and crop types using remote sensing data. IEEE Geoscience and Remote Sensing Letters, 14(5), 778-782. DOI: 10.1109/ LGRS.2017.2681128.

Li, W., Haohuan, F., Le Yu, P., Gong, D.F., Congcong, L. \& Clinton N. (2016). Stacked autoencoder-based deep learning for remote-sensing image classification: A case study of African land-cover mapping. Int. J. Remote Sens., 37(23), 5632-5646. DOI: 10.1080/01431161.2016.1246775.

Li, W., Haohuan, F., Le, Y. \& Cracknell A. (2017). Deep learning based oil palm tree detection and counting for high-resolution remote sensing images. Remote Sensing, 9(1), 22. DOI: 10.3390/rs9010022.

Mahdianpari, M., Salehi, B., Mohammadimanesh, F., Homayouni, S. \& Gill E. (2019). The first wetland inventory map of newfoundland at a spatial resolution of $10 \mathrm{~m}$ using Sentinel-1 and Sentinel-2 data on the Google Earth Engine Cloud Computing Platform. Remote Sensing, 11(1), 43. DOI: $10.3390 / \mathrm{rs} 11010043$ 

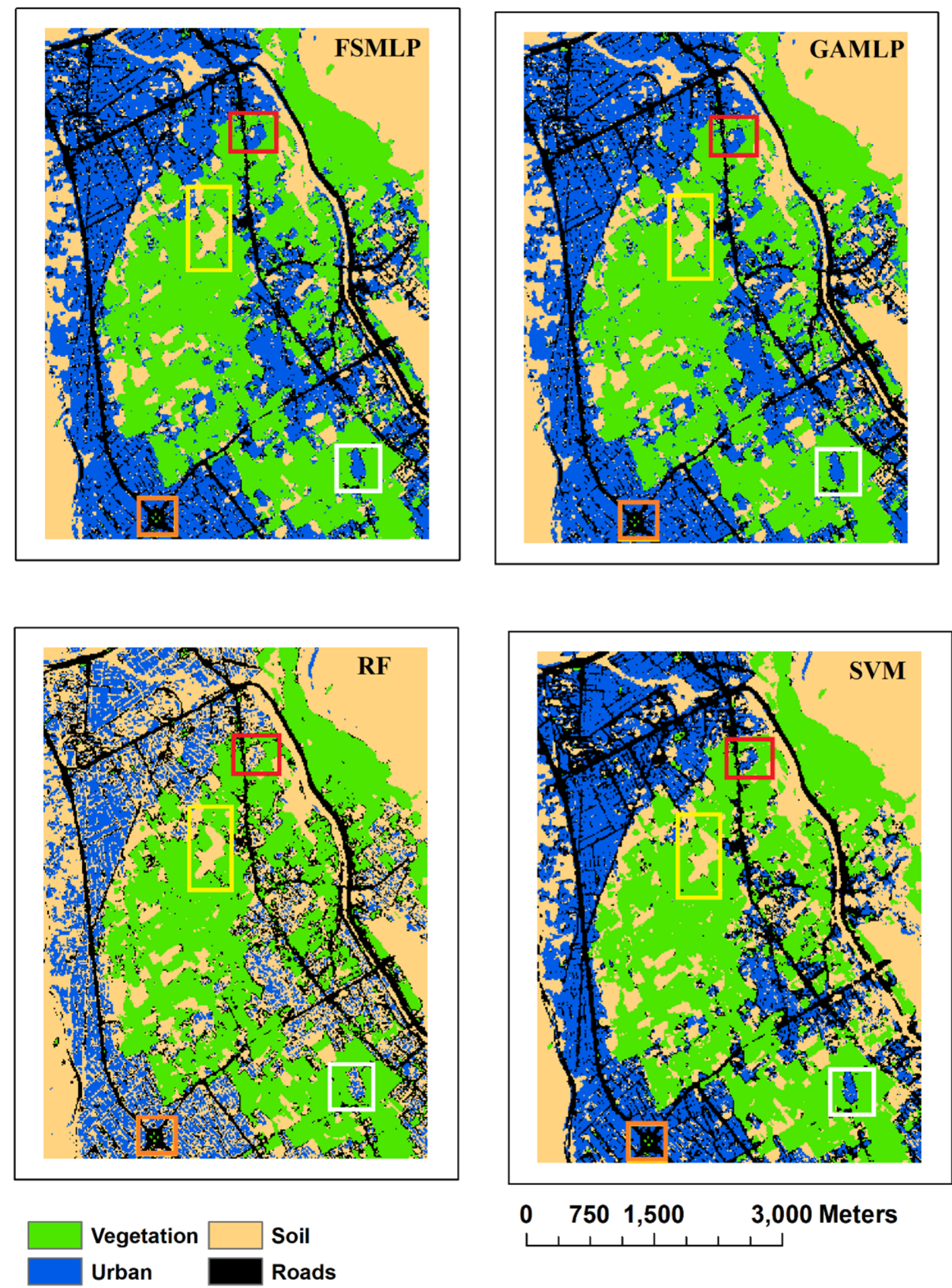

Fig. 8. Final classified maps of the study area in region a for scenario 1 with a spatial resolution of $15 \mathrm{~m}$. 

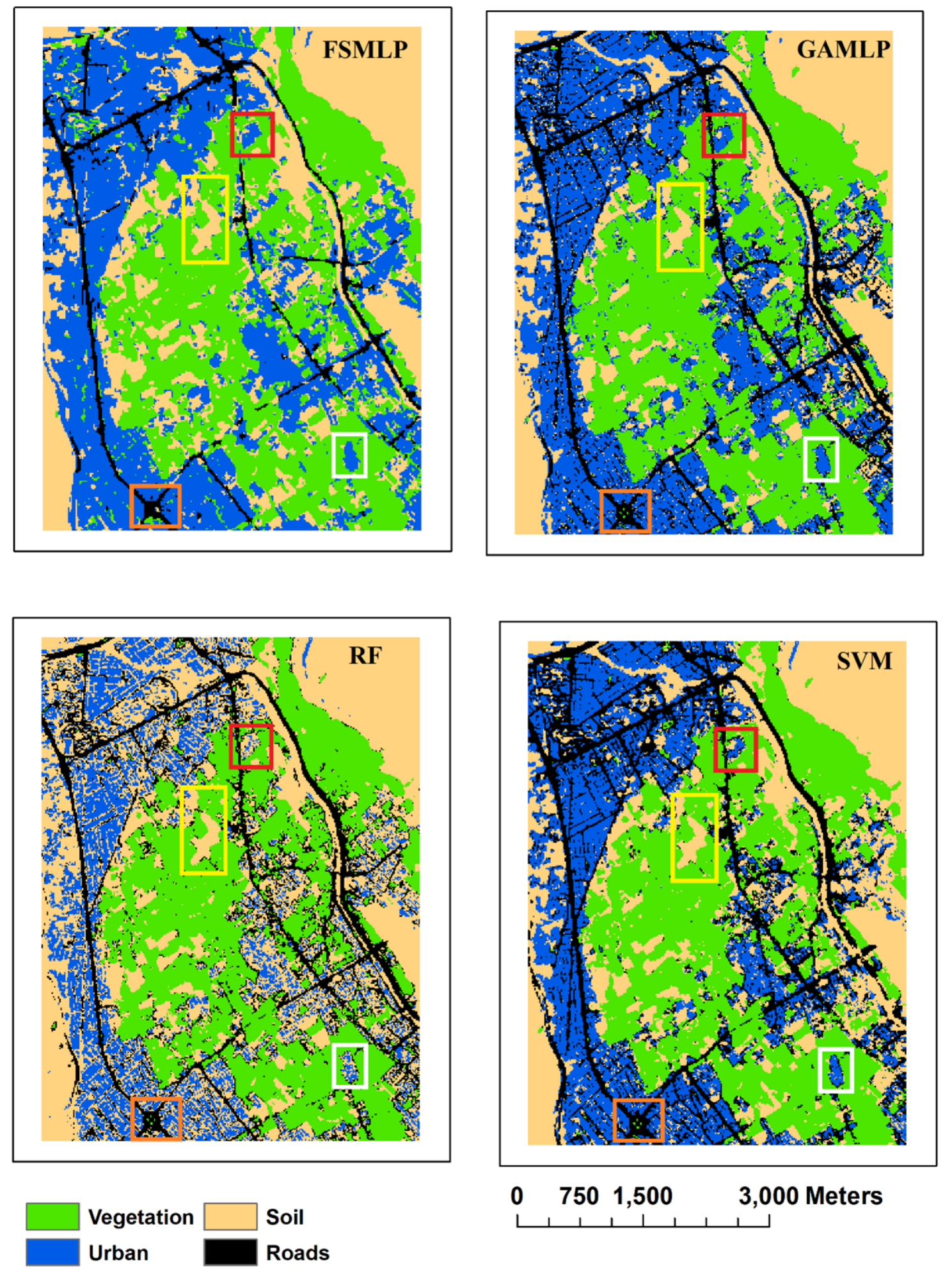

Fig. 9. Final classified maps of the study area in region a for scenario 3 with a spatial resolution of $15 \mathrm{~m}$. 

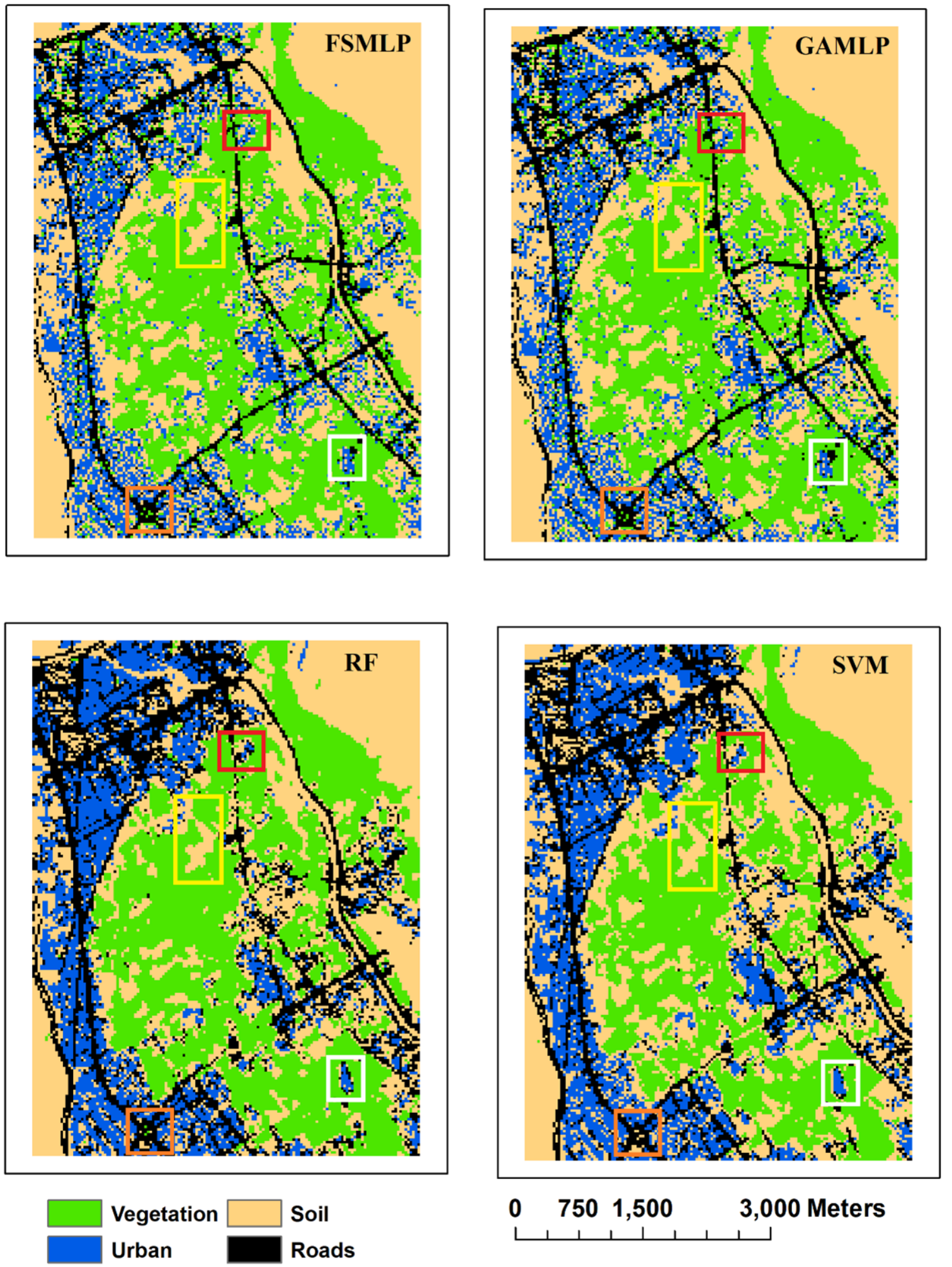

Fig. 10. Final classified maps of the study area in region a for scenario 2 with a spatial resolution of $30 \mathrm{~m}$. 

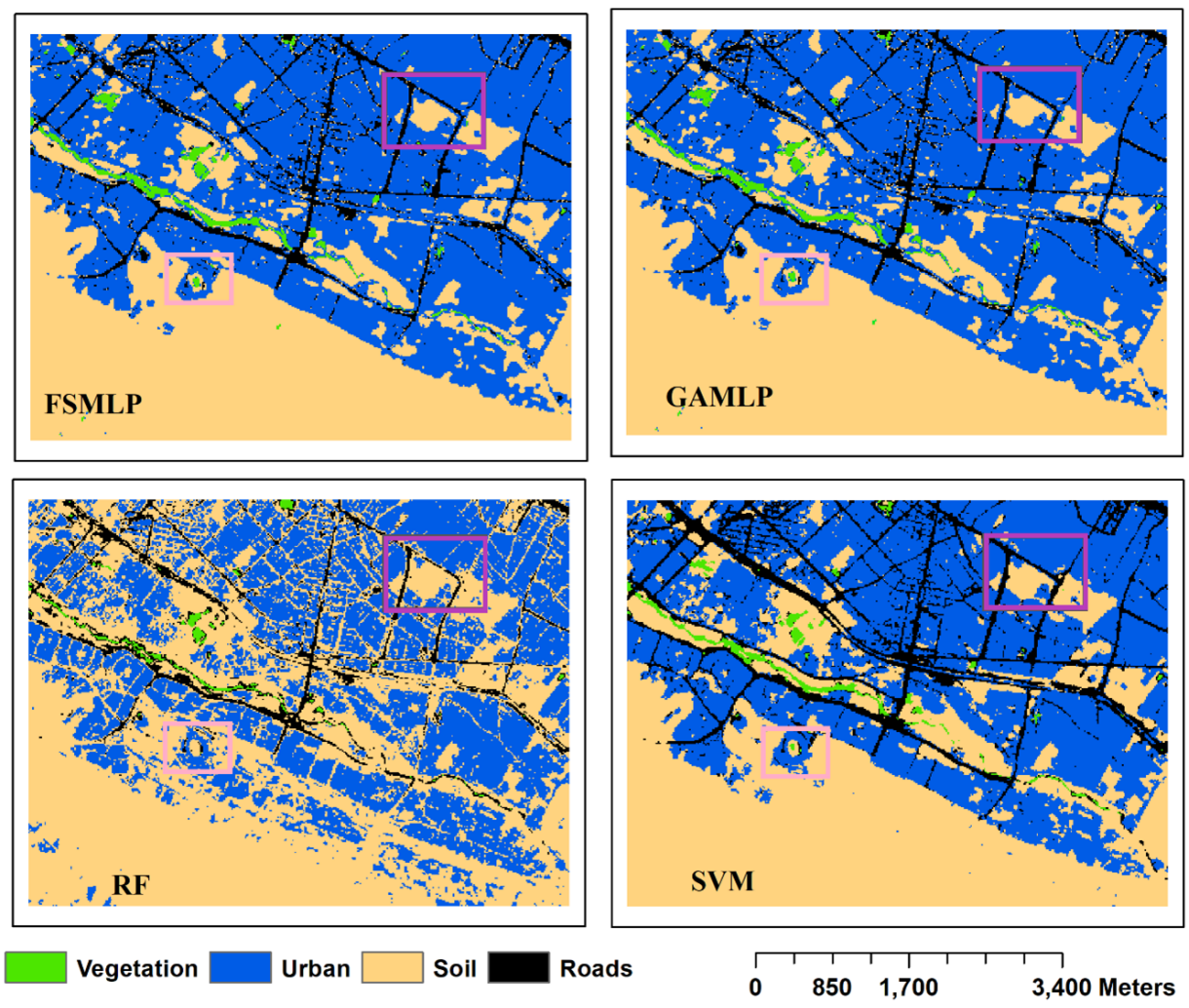

Fig. 11. Final classified maps of the study area in region $\mathrm{b}$ for scenario 1 with a spatial resolution of $15 \mathrm{~m}$.
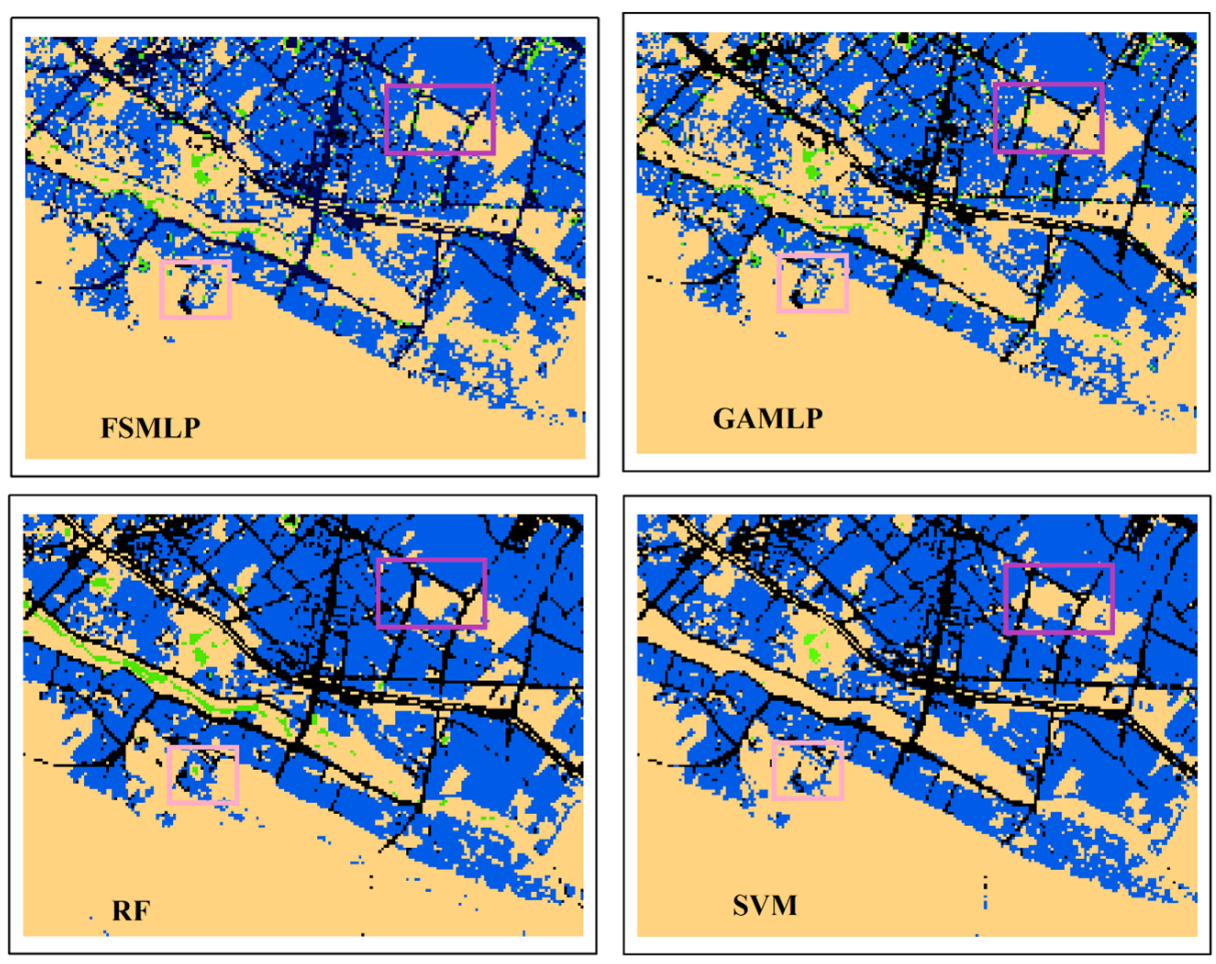

Vegetation

Urban $\square$ Soil

Roads

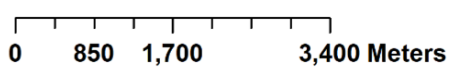

Fig. 12. Final classified maps of the study area in region $b$ for scenario 3 with a spatial resolution of $15 \mathrm{~m}$. 

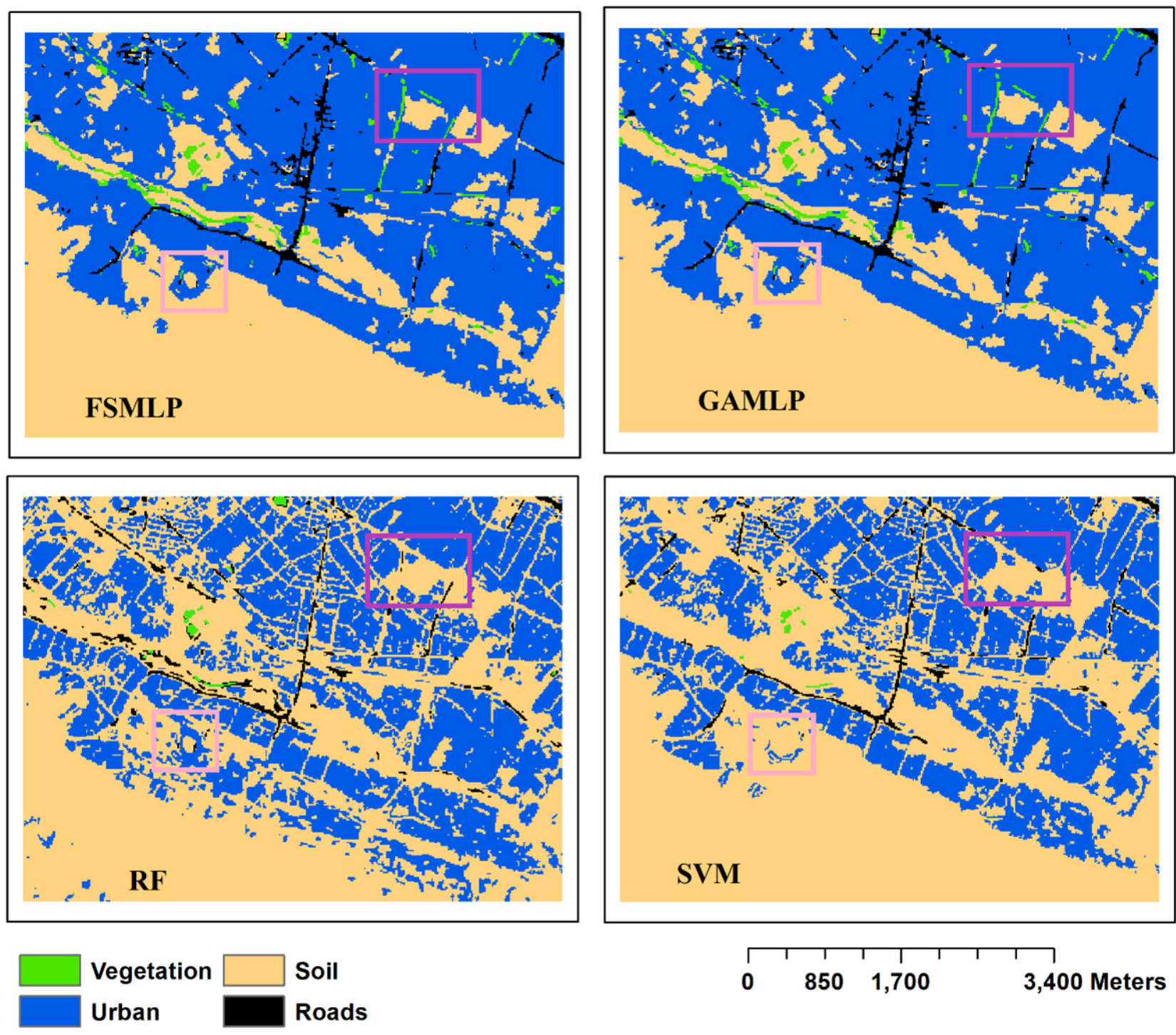

Fig. 13. Final classified maps of the study area in region $\mathrm{b}$ for scenario 2 with a spatial resolution of $30 \mathrm{~m}$.

Mahdianpari, M., Salehi, B., Mohammadimanesh, F. \& Motagh M. (2017) Random forest wetland classification using ALOS-2 L-band, RA DARSAT-2 C-band, and TerraSAR-X imagery. ISPRS Journal of Photogrammetry and Remote Sensing, 130, 13-31. DOI: $10.1016 / \mathrm{j}$.isprsjprs.2017.05.010.

Mansaray, L.R., Wang, F., Huang, J., Yang, L. \& Kanu A.S. (2020). Accuracies of support vector machine and random forest in rice mapping with Sentinel-1A, Landsat-8 and Sentinel-2A datasets. Geocarto International, 35(10), 1088-1108. DOI: 10.1080/10106049.2019.1568586.

Mas, J.F. \& Flores J.J. (2008). The application of artificial neural networks to the analysis of remotely sensed data. Int. J. Remote Sens., 29(3), 617-663. DOI: $10.1080 / 01431160701352154$.

Mohanty, S.P., Hughes, D.P. \& Salathé M. (2016). Using deep learning for image-based plant disease detection. Frontiers in Plant Science, 7, 1419. DOI: $10.3389 /$ fpls.2016.01419.
Mountrakis, G., Jungho, I. \& Ogole C. (2011). Support vector machines in remote sensing: A review. ISPRS Journal of Photogrammetry and Remote Sensing, 66(3), 247-259. DOI: 10.1016/j.isprsjprs.2010.11.001.

Nitze, I., Barrett, B. \& Cawkwell F. (2017). Temporal optimisation of image acquisition for land cover classification with random forest and MODIS Time-series. International Journal of Applied Earth Observation and Geoinformation, 34, 136-146. DOI: 10.1016/ j.jag.2014.08.001.

Rodriguez-Galiano, V.F., Ghimire, B., Rogan, J., Chica-Olmo, M. \& RigolSanchez J.P. (2012). An assessment of the effectiveness of a random forest classifier for land-cover classification. ISPRS Journal of Photogrammetry and Remote Sensing, 67, 93-104. DOI: 10.1016/j.isprsjprs.2011.11.002-

Rogan, J., Franklin, J., Stow, D., Miller, J., Woodcock, C. \& Roberts D. (2008). Mapping land-cover modifications over large areas: a comparison of machine learning algorithms. Remote Sens. Environ., 112(5), 2272-2283. DOI: $10.1016 /$ j.rse.2007.10.004. 
Rouse, J.W., Haas, R.H., Deering, D.W. \& Schell J.A. (1973). Monitoring the vernal advancement and retrogradation (green wave effect) of natural vegetation. Progress Report RSC.

Shao, Y. \& Lunetta R.S. (2012). Comparison of support vector machine, neural network, and CART algorithms for the land-cover classification using limited training data points. ISPRS Journal of Photogrammetry and Remote Sensing, 70, 78-87. DOI: 10.1016/j.isprsjprs.2012.04.001.

Skalský, R., Koco, Š., Barančíková, G., Tarasovičová, Z., Halas, J., Koleda, P., Makovníková, J., Gutteková, M., Tobiášová, E., Gömöryová, E. \& Takáč J. (2020). Land cover and land use change-driven dynamics of soil organic carbon in North-East Slovakian croplands and grasslands between 1970 and 2013. Ekológia (Bratislava), 39(2), 159-173. DOI: 10.2478/ eko-2020-0012.

Vapnik, V. (1982). Estimation of dependences based on empirical data. New York: Springer Verlag. DOI: 10.1007/0-387-34239-7.
Waldrop, M.M. (2016). The chips are down for Moore's Law. Nature, 530(7589), 144-147. DOI: 10.1038/530144a.

Woznicki, S.A., Baynes, J., Panlasigui, S., Mehaffey, M. \& Neale A. (2019) Development of a spatially complete floodplain map of the conterminous United States using random forest. Sci. Total Environ., 647, 942 953. DOI: 10.1016/j.scitotenv.2018.07.353.

Yeom, J., Han, Y. \& Kim Y. (2013). Separability analysis and classification of rice fields using KOMPSAT-2 high resolution satellite imagery. Research Journal of Chemistry and Environment, 17, 136-144.

Zha, Y., Gao, J. \& Ni S. (2003). Use of normalized difference built-up index in automatically mapping urban areas from TM imagery. Int. J. Remote Sens., 24(3), 583-594. DOI: 10.1080/01431160304987. 